\title{
Surgical strategies for liver cell adenoma
}

\author{
Frederic E. Eckhauser, Lisa M. Colletti, and James A. Knol \\ Division of Gastrointestinal Surgery, Department of Surgery, University of Michigan Medical Center, 1500 E. Medical Center Drive, Ann \\ Arbor, MI 48109-0331, USA
}

Key words: benign liver tumors, hepatic adenoma, liver cell adenoma, focal nodular hyperplasia

\section{Intreduction}

Liver cell adenomas (LCA) are benign tumors that occur primarily in women with a history of oral contraceptive use. While rare, LCA are clinically important because of their propensity for sudden, unpredictable hemorhage or malignant transformation. Differentiation from focal nodular hyperplasia or hepatocellular carcinoma may be difficult even with sophisticated imaging modalities. Current management guidelines are based on the presence or absence of symptoms, the certainty of diagnosis, and the response, if any, to withdrawing putative growth stimuli. Complete excision, sparing as much normal liver as possible, is generally recommended, except in patients with adenomatosis. This may require anatomical resection (either lobectomy or segmentectomy), local wedge excision, or enucleation. Rarely, orthotopic liver transplantation may be required in patients with large, centrally placed lesions, recurrence following previous resection(s), or adenomatosis. Nonsurgical ablation techniques such as interstitial radio-frequency or laser hyperthermia and cryotherapy may also be useful in selected patients.

\section{Discussion}

Benign liver tumors are fairly common, with an estimated autopsy incidence of $1 \%$ or $2 \% .^{1}$ Those encountered most frequently in clinical practice include

Offprint requests to: F.E. Eckhauser

Received for publication on July 17, 1995; accepted on Oct. 12,1995 hemangioma, focal nodular hyperplasia (FNH), and liver cell adenoma (LCA). Hemangiomas differ from FNH and LCA in many respects. They are congenital rather than neoplastic in origin, and there are no well documented reports of malignant transformation. Compared to FNH and LCA, which are solid tumors, hemangiomas are composed microscopically of dilated vascular sinuses lined with endothelium. Increase in diameter over time occurs in a minority of patients and is usually not associated with the onset or worsening of symptoms. Reports of significant enlargement during pregnancy or in patients taking oral contraceptives are largely anecdotal, suggesting that the growth of hemangiomas may not be entirely hormone-dependent. A definitive diagnosis can be made with $90 \%$ sensitivity and accuracy with either magnetic resonance imaging (MRI) or single-photon emission computed tomography (SPECT), using technetium $99 \mathrm{~m}$-labeled red blood cells. ${ }^{2}$ Based on these observations, many centers now advocate conservative therapy, and limit surgery to patients with incapacitating symptoms or life-threatening complications. ${ }^{3,4}$ When surgery is indicated, enucleation is preferred to formal hepatic resection, and can be accomplished safely with minimal disturbance of liver function and preservation of normal functioning parenchyma. 5,6

FNH and LCA are separate and distinct benign lesions that differ in etiology, morphology, and natural history. Unfortunately, treatment dilemmas occur with some regularity because FNH and LCA may be indistinguishable based on clinical, pathologic, and radiographic features.

Patients with LCA are generally women of childbearing age with a history of oral contraceptive use. Little was known about these unusual tumors until 1973, when Baum published a landmark paper establishing a clear association betwen oral contraceptives and the development of LCA. ${ }^{7}$ We have since learned that LCA arising in oral contraceptive users tend to be 
larger and are associated with higher rates of intratumoral and intraperitoneal hemorrhage that those occurring in nonusers. ${ }^{8}$ Additional risk factors include androgenic steroids, Type la glycogen storage disease, and insulin-dependent diabetes mellitus. Dietary therapy aimed at correcting abnormal levels of insulin, glucagon, and glucose in patients with LCA and Type Ia glycogen storage disease may result in tumor regression, suggesting that insulin and/or glucagon may be putative growth stimuli.

Although the populations of patients developing LCA and FNH are similar, there is a lower correlation with oral contraceptive use in patients with FNH. The mode of presentation also differs for the two lesions. Nearly $90 \%$ of FNH cases are discovered incidentally at autopsy, during operations for unrelated indications, or on routine physical examination..$^{9,10}$ Patients with FNH may complain of chronic, nonspecific upper abdominal discomfort, but rarely present with acute symptoms or abdominal findings. In contrast, up to $80 \%$ of patients with LCA are symptomatic. ${ }^{8-10}$ Of these patients, onehalf present with symptoms related to mass effect (pain, early postprandial satiety, etc), and the remainder present with symptoms dur to tumor rupture or hemorrhage. ${ }^{8}$ Constant or premonitory symptoms are uncommon among patients with acute symptoms and rarely aid in establishing a preoperative diagnosis.

Among patients with ruptured LCA there is a wide spectrum of presentation and treatment. Spontaneous intratumoral hemorrhage may cause acute right upper quadrant pain that mimics biliary colic. LCA are prone to bleed because they consist of multiple sinusoids of thin-walled capillaries supplied by arterial blood under high pressure. In addition, they contain little connective tissue support, which allows blood to disperse extensively throughout the tumor. ${ }^{11}$ Nearly two-thirds of patients presenting with acute abdominal pain and hypotension require emergency surgery to control bleeding. ${ }^{12}$ In this setting, the average mortality rate is approximately $6 \%$, but rates approaching $20 \%$ have been reported. ${ }^{12,13}$ While the size of an adenoma generally predicts the likelihood of hemorrhage, the severity of bleeding does not necessarily correlate with the size of the tumor. The remainding one-third of patients develop stable hematomas and can be monitored shortterm in an intensive care unit setting. One should not be lulled into a false sense of security in these patients if hemorrhage ceases spontaneously and the lesion appears stable on CT or ultrasound. Because of the sudden and unpredictable threat of repeated hemorrhage, all patients with acutely symptomatic LCA should ultimately undergo some definitive form of surgical treatment.

Treatment dilemmas arise chiefly because of the difficulty in distinguishing LCA from other benign liver tumors and from primary or metastatic liver cancer. Biochemical liver function tests are not helpful. Ultrasonographic features are nonspecific and CT has a low positive predictive value that precludes routine clinical use. ${ }^{14}$ The limited efficacy of US and CT may be partly explained by the fact that these lesions exhibit imaging characteristics that are similar to those of normal surrounding hepatic parenchyma. Characteristic findings such as a stellate scar (FNH) and intralesional hemorrhage or necrosis (LCA) are distinctly uncommon. Angiography accurately defines hepatic vascular anatomy but lacks specificity. ${ }^{15}$ Nuclear scintigraphy with technetium-labelled sulfur colloid has been used to differentiate LCA from FNH, based on the premise that FNH, which contains cells, will selectively take up isotope and appear as a "hot" area, while LCA, which do not contain cells, will appear as a void or filling defect. However, scintigraphic and pathologic data from one recent retrospective study of 13 proven cases of LCA demonstrated histologic evidence of cells in all cases, and uptake of technetium- $99 \mathrm{~m}$ sulfur colloid in 3 cases (23\%). ${ }^{15}$ Conversely, focal defects can be demonstrated in approximately $30 \%$ of patients with proven $\mathrm{FNH}^{10}$ Isotope scans are further limited because the voids thought to be characteristic of LCA can also occur with primary or metastatic liver cancer and hemangiomas.

At present no single radiologic modality has sufficient specificity to be diagnostic in this setting. CT and, more recently, MR provide descriptive information regarding the number and location of lesions and may suggest the diagnosis of FNH or LCA based on the presence of a central avascular scar and intratumoral hemorrhage or necrosis, respectively. In our experience and that of others, scintigraphy should also be obtained to provide data relevant to diagnosis and treatment. In general, a solitary mass that exhibits normal or increased uptake of radio-colloid will prove to be FNH, especially if identified in an asymptomatic young woman without liver disease. Complementary findings of homogeneity and/ or slight hypodensity and lack of intratumoral hemorrhage on CT and hypervascularity with an intense capillary stain on angiography will help to confirm the diagnosis of FNH and should be sufficient for clinical management in the absence of histopatholgy.

FNH has a benign prognosis and can be managed conservatively in asymptomatic patients once the diagnosis has been established with reasonable certainty. Progression of symptoms or development of complications in patients treated expectantly is extremely uncommon. When indicated, excision can be safely recommended, with minimal reported operative mortality and morbidity..$^{10}$ Following successful excision of $\mathrm{FNH}$, patients can be reassured that recurrence is unlikely even if they resume oral contraceptives or become pregnant. 
Patients with suspected or proven LCA require a different strategy. The majority of acutely symptomatic patients will require excision, frequently in the absence of a hisopathologic diagnosis. In asymptomatic patients, recommendations regarding excision versus expectant therapy are colored by concerns regarding potential malignant transformation to carcinoma. Unfortunately there is remarkably little information regarding the natural history of unresected LCA. In a recent review of the literature, Foster and Berman ${ }^{16}$ identified only five instances of probable transformation from LCA to cancer, including four patients taking oral contraceptives at the time of diagnosis. Despite cessation of oral contraceptives and apparent tumor stabilization or regression, all patients developed cancer within 2-7 years. They also analyzed descriptions of 22 additional patients with single or multiple LCAs that were either biopsied or incompletely resected and followed for periods ranging from 3 months to 10 years. Tumor involution occurred in eight of nine patients $(89 \%)$ following cessation of oral contraceptives, one patient underwent resection for tumor regrowth, and none of the patients experienced rupture or developed cancer.

The risk of transformation from LCA to cancer has traditionally been viewed as a compelling argument for excision, tempered partially be the risks of major hepatic resection, especially in young, healthy, and otherwise aymptomatic patients. This risk may be somewhat overstated but is arguably real and cannot be discounted. Given the decreased risk of hepatic resection when performed by skilled and experienced surgeons, most experts now recommend resection of all solitary LCA, even if they involute following cessation of oral contraceptives. Patients with large central tumors or multiple tumors who undergo incomplete resection must be followed for decades with serial imaging studies and should abstain indefinitely from oral contraceptives, anabolic steroids, and other putative growth stimuli. Serial measurements of alfa-fetoprotein may be helpful in detecting malignant change. ${ }^{16}$

Multiple adenomas occur in $12 \%-30 \%$ of patients and present special surgical problems. ${ }^{9,11}$ Children with Type I or III glycogen storage disease and patients receiving androgenic steroids are prone to develop multiple LCA with attendant risks of hemorrhage or malignant transformation. Adenomatosis is rare disorder characterized by the occurrence of multiple $(>10)$ adenomas in the liver, Adenomatosis and liver cell adenoma differ in many important chracteristics and may, therefore, represent distinct entities., ${ }^{9,17}$ There is no female predominance in adenomatosis and oral contraceptives are generally not implicated as etiologic factors. In addition, intratumoral or intraperitoneal hemorrhage, which occurs with both conditions, may be more common with adenomatosis. The lesions of adenomatosis vary in size from 0.5 to $>10 \mathrm{~cm}$, are distributed uniformly throughout both lobes of the liver, and histologically consist of unencapsulated aggregations of small-sized hepatocytes that contain no portal triads. ${ }^{17}$ In patients presenting with acute hemorrhage, surgery should consist of enucleation or resectional debridement, sparing as much normal parenchyma as possible. Spontaneous malignant transformation has been demonstrated in at least one patient 5 years after the original diagnosis of adenomatosis. ${ }^{9}$ In view of this malignant potential, adenomatosis should perhaps be considered as an indication for liver transplantation. However, the rarity of the complication may not justify this aggressive an approach.

The surgical approach to LCA has evolved considerably over the past several decades. Despite advances in radiologic imaging and nuclear scintigraphy, accurate differentiation of LCA from other bengin or malignant liver lesions may be difficult. In patients with acute symptoms of intratumoral or intraperitoneal hemorrhage, surgical intervention is warranted in all cases, regardless of whether the bleeding ceases spontaneously. Increase in tumor size, indeterminate diagnosis, and progression of mass-related symptoms are additional indications for surgical excision. Until recently, formal hepatic resection was considered a hazardous procedure, with reported mortality rates of $4 \%-20 \% .{ }^{18-20}$ The major causes of perioperative mortality associated with hepatic resection are uncontrollable hemorrhage or injury to the blood supply or biliary ducts supplying the unresected segments of liver. There is a also strong correlation between operative blood loss and the incidence and severity of postoperative complications.

Formal hepatic resection is currently a safe procedure with minimal mortality and complication rates of $<10 \%{ }^{9,11,21}$ Several factors have contributed to improved results, including a better understanding of liver anatomy, expanded use of intraoperative ultrasonography, and technical refinements that facilitate controlled hepatic resection with minimal blood loss. Based on prior studies of hepatic anatomy, we now understand that the liver can be functionally divided into eight segments based on the distribution of the portal pedicles and the location of the hepatic veins. Each of these segments can theoretically be resected while maintaining the integrity of the remaining segments. $^{22}$ This classification provides the surgeon with a powerful tool and is a prerequisite for performing safe liver surgery. Increased availability and familiarity with intraoperative ultrasound, especially B-mode or realtime scanning, allows the surgeon to precisely identify internal vascular architecture, identify unsuspected pathology, and plan sectoral or segmental resections based on portal and hepatic venous structures. Useful infor- 
mation that alters operative strategy can be obtained in up to $30 \%$ of patients using this technology. ${ }^{23}$

Two distinct techniques of liver resection have been described. Resection with primary vascular control was first described by Lortat-Jacob and colleagues in the early $1950 \mathrm{~s}^{24}$ and consists of ligating and dividing portal pedicles and draining hepatic veins before transecting the parenchyma. Advantages include reduction of bleeding and sharp demarcation of the devascularized portion of liver to be resected. The major disadvantage is massive and often uncontrollable bleeding from a torn hepatic vein or the vena cava and the attendant potential for air embolism. An alternative approach, popularized by Bismuth, ${ }^{22}$ consists of temporary inflow occlusion combined with primary parenchymal transection in the plane of lobar division. Using this technique, the portal elements and hepatic veins are secured as they traverse the plane of dissection within the substance of the liver. Primary parenchymal dissection reduces the potential risk of injury to inflow or outflow vessels, but may be associated with increased bleeding and usually requires either partial or total vascular exclusion of the liver.

Hepatic vascular exclusion facilitates resection in patients with large or posterior tumors, in whom the risk of massive bleeding or air embolism is appreciable. This technique was originally described by Heaney and Jacobson in $1975^{25}$ and has been summarized recently in an excellent article by Huguet et al. ${ }^{26}$ This approach entials clamping of the portal inflow vessels and the inferior vena cava above and below the liver prior to parenchymal transection. Accessory or replaced hepatic arteries must be identified and clamped to prevent troublesome bleeding from the plane of dissection. Occluding clamps are placed in a specific order to minimize hepatic venous congestion: portal pedicle followed by infrahepatic and suprahepatic portions of the inferior vena cava, respecitvely. Following resection, the infrahepatic vena cava clamp is partially released to aid in identifying vessls along the cut surface of the liver that might require suture ligation. The suprahepatic and portal pedicle clamps are then removed, and the perihepatic space is drained widely with silicone drains exited through the side or flank.

The efficacy and safety of hepatic vascular exclusion have been documented in serveral recent series with excellent metabolic tolerance of the liver for periods of up to $1 \mathrm{~h} .{ }^{27,28}$ Significant reduction of intraoperative bleeding, associated coagulation problems, and postoperative complications have been reported using this technique. ${ }^{27}$ Data from our institution indicates that the use of this technique markedly decreases intraoperative blood loss in patients who undergo resection or enucleation of liver cell adenomas. ${ }^{29}$ One must keep in mind that attention to technical details is critical for achieving total vascular exclusion. Improper positioning of the caval clamps or failure to identify an accessory or replaced hepatic artery will lead to hepatic venous congestion, troublesome bleeding that impairs visualization of the operative field, and possible hemodynamic compromise for the patient. When used properly, this technique reduces operative blood loss and the health risks associated with blood transfusion.

Formal hepatic resection may not be necessary once the histopathologic diagnosis of LCA has been confirmed and the specter of malignancy has been dispelled. This is especially important, since features of LCA and hepatocellular carcinoma can occasionally coexist within the same lesion. ${ }^{9}$ Most single adenomas can be enucleated, even those located posteriorly or within the substance of the liver. The major decision is whether to employ partial (inflow) occlusion or total vascular exclusion prior to enucleation. Enucleation of hepatic hemangiomas has been well described, is both safe and effective, and has largely replaced hepatic resection for treatment of these benign tumors. ${ }^{5,6}$ The technique is relatively straightforward. Once vascular control has been achieved, the liver capsule is incised several millimeters beyond the periphery of the tumor. A cleavage plane or sheath of compressed liver tissue that defines the border between the hemangiomatous tissue and the normal liver is easily defined and guides further dissection, using either the surgeon's finger or the ultrasonic aspirator. We prefer to use the ultrasonic aspirator, which aids in identifying and skeletonizing major bile ducts and vascular structures for careful ligation and division. Careful dissection in the cleavage plane maintains a relatively bloodless field and facilitates complete removal of the tumor. Once the tumor has been enucleated, the resulting defect can be managed by external catheter drainage alone or combined with mobilization of a flap of vascularized omentum to obliterate the cavity. Enucleation is equally applicable to all benign tumors of the liver and has already been used sucessfully for treatment of patients with liver cell adenomas. ${ }^{29}$ This technique is particularly useful for patients with two or three adenomas in whom formal resection would sacrifice an excessive amount of normal parenchyma. In addition to being useful for benign liver tumors, enucleation has also been used successfully to treat hydatid cysts of the liver..$^{30}$

In addition to formal hepatic resection and enucleation, other forms of surgical therapy are also available to treat benign tumors of the liver. Interstitial hyperthermia employing radiofrequency electrocautery has been used successfuly to treat one patient with an LCA who refused more traditional treatment. ${ }^{31}$ The extent of necrosis induced by the needle is exactly predictable based on the size of the needle, the temperature of the needle tip, and the exposure time. The 
procedure can be accomplished laparoscopically and is most useful for peripherally-situated lesions. However, based on experience with thermal ablation of hepatocellular cancers, deeper adenomas should be safely accessible with ultrasound guidance. ${ }^{32}$ Cryoablation has also been used successfully to treat LCA, and may be especially useful for centrally located tumors that would otherwise require trisegmentectomy. ${ }^{33}$ Finally, orthotopic liver transplantation (OLT) may be necessary in symptomatic patients with potentially lifethreatening complications if tumor size or location preclude safe resection, or if prior resection has failed to control symptoms. OLT will rarely be needed for benign liver lesions. In one series of 3239 patients undergoing OLT, only 12 patients $(0.37 \%)$ required transplantation for benign, symptomatic neoplasms. ${ }^{33}$ The indications for OLT in five of the six adenoma patients who required urgent transplantation included sustained growth and recurrent intratumoral or intraperitoneal hemorrhage. Long-term outcome following OLT was excellent, with a reported median survival of 88 months. While seemingly extreme, OLT may be the treatment of choice for large, life-threatening benign tumors that preclude more conventional forms of treatment.

\section{References}

1. Henson SW Jr, Gray HK, Dockerty MB (1956) Benign tumors of the liver. Surg Gynecol Obstet 102:23-30

2. Birnbaum BA, Weinreb IC, Megibow AI (1990) Definitive diagnosis of hepatic hemangiomas: MR imaging versus Tc- $99 \mathrm{~m}$ labeled red blood cell SPECT. Radiology 176:95-102

3. Sinanan MN, Marchiorio T (1989) Management of cavernous hemangioma of the liver. Am J Surg 157:519-522

4. Farges O, Daradkeh S, Bismuth H (1995) Cavernous hemangiomas of the liver: Are there any indications for resection? World J Surg 19:19-24

5. Alper A, Ariogul O, Emre A, Uras A, Okten A (1988) Treatment of liver hemangiomas by enucleation. Arch Surg 123:660-661

6. Baer HU, Dennison AR, Mouton W, Stain SC, Zimmerman A, Blumgart LH (1992) Enucleation of giant hemangiomas of the liver: Technical and pathological aspects of neglected procedure. Ann Surg 216:673-676

7. Baum JK, Bookstein JJ, Holz F, Kein EW (1973) Possible association between benign hepatomas and oral contraceptives. Lancet II: $926-929$

8. Shortell CK, Schwartz SI (1991) Hepatic adenoma and focal nodular hyperplasia. Surg Gynecol Obstet 173:426-431

9. Kerlin P, Davis GL, McGill DB, Weiland LH, Adson MA, Sheedy PF II (1983) Hepatic adenoma and focal nodular hyperplasia: Clinical, pathoogic and radiologic features. Gastroenterology 84:994-1002
10. Nagorney DM (1995) Benign hepatic tumors: Focal nodular hyperplasia and hepatocellular adenoma. World J Surg 19:13-18

11. Leese T, Farges O, Bismuth H (1988) Liver cell adenomas: A 12year surgical experience from a specialist hepatobiliary unit. Ann Surg 208:558-564

12. Flowers BF, McBurney RP, Vera SR (1990) Ruptured hepatic adenoma: A spectrum of presentation and treatment. Am Surg 56:380-383

13. Klatskin G (1977) Hepatic tumors: Possible relationship to use of oral contraceptives. Gastronenterology 84:994-1002

14. Mathieu D. Bruneton JN, Drouillard J, Pointreau CC, Vasile N (1986) Hepatic adenomas and focal nodular hyperplasia: Dynamic CT study. Radiology 160:53-58

15. Lubbers Pr, Pos PR, Goodman ZD, Ishak KG (1987) Accumulation of technitium- $99 \mathrm{~m}$ sulfur colloid by hepatocellular adenoma: Scintigraphic-pathologic correlation. Am J Roentgenol (AJR) 148:1105-1108

16. Foster JH, Berman MM (1994) The malignant transformation of liver cell adenomas. Arch Surg 129:712-717

17. Flejou J-F, Barge J, Menu Y, Degott C, Bismuth H, Potet F, Benhamou J-P (1985) Liver adenomatosis: An entity distinct from liver adenoma. Gastroenterology 89:1132-1139

18. Thompson H, Tompkins R, Longmire W (1983) Major hepatic resection: A 25-year experience. Ann Surg 197:375-388

19. Iwastsuki S, Shaw B, Starzl T (1983) Experience with 150 liver resections. Ann Surg 197:247-253

20. Adson MA, Weiland LH (1981) Resection of primary solid hepatic tumors. Am J Surg 141:18-21

21. Iwatsuki S, Toto S, Starzl T (1990) Excisional therapy for benign hepatic lesions. Surg Gynecol Obstet 171:240-248

22. Bismuth $H$ (1982) Surgical anatomy and anatomical surgery of the liver World Surg 6:3-9

23. Castaing D, Edmond J, Bismuth H, Kuntslinger F (1985) Utility of intraoperative ultrasound in the surgical mangement of liver tumors. Ann Surg 204:600-605

24. Lortat-Jacob JL, Robert HG, Henry C (1952) Un cas d'hepatectomie droite regle. Memoires del'Academie de Chirugie $78: 244-251$

25. Heaney JP, Jacobson A (1975) Simplified control of upper abdominal hemorrhage from the vena cava. Surgery 78:138-141

26. Huguet C, Addario-Chieco P, Gavelli A (1992) Technique of hepatic vascular exclusion for extensive liver resection. Am J Surg 163:602-605

27. Ryan JA, Faulkner J (1989) Liver resection without blood transfusion. Am J Surg 157:472-475

28. Bismuth H, Castaing D, Garden J (1989) Major liver rescetion under total vascular exclusion. Ann Surg 209:211-218

29. Eckhauser FE, Knol JA, Raper SE, Thompson NW (1994) Enucleation combined with hepatic vasculat exclusion is a safe and effective alternative to hepatic resection for liver cell adenoma. Am Surg 60:466-472

30. Belli L, Favero E, Marni A (1983) Resection vs pericystectomy in the treatment of hydatidosis of the liver. Am J Surg 145:239-242

31. Buscarini L, Rossi S, Fornari F, DiStasi M, Buscarini E (1995) Laparoscopic ablation of liver adenoma with radiofrequency electrocautery. Gastrointest Endosc 41:68-70

32. Schwartz SI, Henderson JM, Jones RS, Nagorney DM, Sitzmann JV (1995) Symposium: Management of benign liver tumors. Contemp Surg 46:269-280

33. Tepetes K, Selby R, Webb M, Maraiaga JR, Iwatsuki S, Starzl T (1995) Orthotopic liver transplantation for benign hepatic neoplasms. Arch Surg 130:153-156 